\title{
PREVAILING SATURATION FLOW RATE FOR LANE GROUPS IN AN URBAN AREA
}

\author{
Stephen Arhin ${ }^{1}$, Melissa F. Anderson ${ }^{2}$, Regis Stinson ${ }^{3}$, Asteway Ribbiso ${ }^{4}$ \\ 1,2,3,4 Department of Civil and Environmental Engineering, Howard University, Washington, DC, 20059, \\ United States of America
}

Received 25 November 2015; accepted 5 April 2016

\begin{abstract}
This study focused on determining the prevailing Saturation Flow Rate (SFR) for specific lane groups in an urban area: District of Columbia (DC). The lane groups considered were Through (T), shared Through and Right (TR), shared Through and Left (TL), and exclusive Left turn (L) lane groups. These SFR values could then be used to calculate the local base SFR. The study determined the prevailing SFR for these lane groups based on data collected at 81 intersections. The hypothesis that the mean SFRs for all the lane groups are different was tested at a $5 \%$ level of significance. From the results, the mean prevailing SFR for the T, TR, TL and $L$ lane groups were 1,559, 1,461, 1,566 and 1,460 vphpl respectively. Those prevailing SFRs can be used for planning analyses in the District of Columbia. The results also indicated that these mean prevailing SFRs are statistically similar at $95 \%$ confidence interval. Based on the results, a local base SFR for the City can be determined for each lane group.
\end{abstract}

Keywords: prevailing saturation flow rate, District of Columbia, lane group.

\section{Introduction}

Saturation Flow Rate (SFR) is a measure of the maximum flow rate of traffic in a specific lane group on an approach to a signalized intersection. SFR is used extensively in planning and operational analysis, and design of signalized intersections. Assuming that an intersection's approach signal could stay green for an entire hour, and the traffic is as dense as it could reasonably be expected, then the maximum number of vehicles that would pass through the approach during that time is defined as the SFR. Therefore, the SFR for a signalized intersection lane group can be defined in broad terms as the maximum number of passenger car units (pcu) per hour of green that flow through a particular intersection lane group. SFR is integrated into several software programs for traffic operation and planning evaluations, such as the Highway Capacity Software (HCS), Synchro, and Transyt-7F. The calculation of vehicle delays and hence the Level of Service (LOS) for intersections relies on reasonable values of SFR. SFR is a critical factor used in LOS analyses for signalized intersections via the methods of the Highway Capacity Manual (HCM).

SFR depends on several roadway and traffic conditions (parking, lane configuration, lane width, presence of heavy vehicles, traffic behavior, number of lanes, traffic volumes, approach grade, area type, etc.) and can vary substantially from one jurisdiction to

${ }^{1}$ Corresponding author: saarhin@howard.edu 
another. A review of the literature indicates that the following factors influence the magnitude of the SFR:

- Traffic composition - different types of vehicles, motorized and non-motorized, with different operating performances, such as signal timing;

- Driver behavior - poor lane discipline and observation of traffic signals;

- Public transport - varied mix of bus types, bus stop locations, and driving styles;

- Roadside activity - roadside land uses generate parking and non-transport activities that reduce effective lane width;

- $\quad$ Speed limits;

- Gradient;

- Right-turn and left-turn lanes;

- Number of through lanes;

- Area type - Central Business District (CBD) or non-CBD.

In conducting LOS analyses, a base SFR is typically used. Some jurisdictions have determined a local based SFR that is representative of their respective regions. Determining a prevailing SFR for each lane group is the first task undertaken in the determination of a local base SFR. The prevailing SFR is determined by using the average headway obtained from field measurements and defined as the number of vehicles per hour per lane through a specific intersection approach and lane group.

This study was aimed at determining the average prevailing SFR for four different lane groups in the District of Columbia. The four types of lane groups considered in this study are: through $(\mathrm{T})$, shared through and right (TR), shared through and left (TL), and exclusive left turn (L). The average prevailing SFRs for these lane groups can be used to compute the local base SFR. The hypothesis that the average prevailing SFRs for all four lane groups are the same was tested at $5 \%$ level of significance.

\section{Literature Review}

Saturation flow rate is defined as the flow rate, in vehicles per hour, that can be accommodated by a lane group, assuming that the green phase was displayed 100 percent of the time, (that is, $\mathrm{g} / \mathrm{C}=1.0$ ), where $g$ is the effective green time and $C$ is the cycle length (Highway Capacity Manual, 2010). It is computed by Eq. (1):

$s=\frac{3600}{h}$

where,

$s=$ saturation flow rate (vphpl)

$h=$ saturation headway (s/veh)

The default saturation headway is $1.9 \mathrm{~s}$, which corresponds to a default base saturation of 1900 pc/h/ln (Highway Capacity Manual, 2010). The idealized saturation flow rate is shown in Fig. 1.

The saturation flow rate for each lane group is computed by Eq. (2):

$s=s_{o} N f_{W} f_{H V} f_{g} f_{p} f_{b b} f_{a} f_{L U} f_{R T} f_{L T} f_{L p b} f_{R p b}$ 


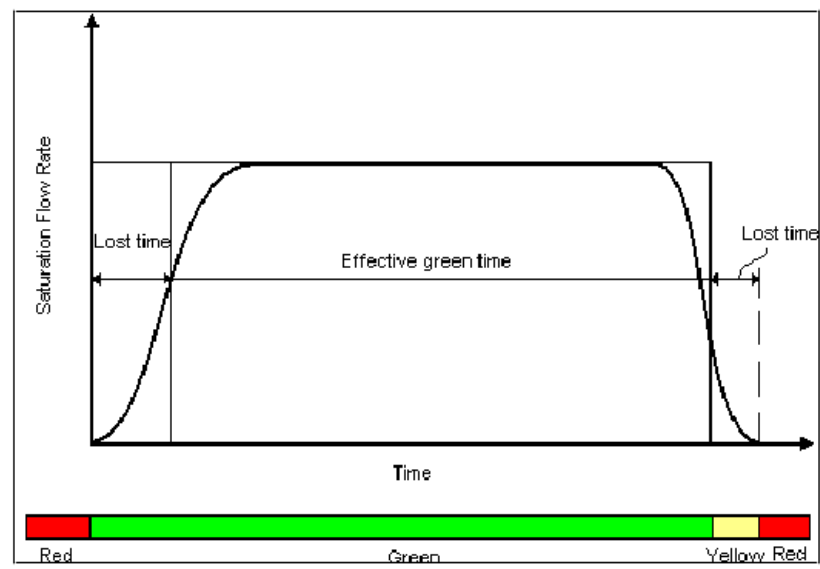

Fig. 1.

Traffic Flow during the Green Period from a Saturated Approach

Source: Highway Capacity Manual (2010)

where:

$s=$ saturation flow rate for subject lane group (vphgpl)

$S_{O}=$ ideal saturation flow rate per lane $(\mathrm{pc} / \mathrm{h} / \mathrm{ln})$

$\mathrm{N}=$ number of lanes per group

$f_{W}=$ adjustment factor for lane width

$f_{H V}=$ adjustment factor for heavy vehicles in traffic stream

$f_{g}=$ adjustment factor for approach grade

$f_{p}=$ adjustment factor for existence of a parking lane and parking activity adjacent to lane group

$f_{b b}=$ adjustment factor for blocking effect of local buses that stop within intersection area $f_{a}=$ adjustment factor for area type

$f_{L U}=$ adjustment factor for lane utilization

$f_{R T}=$ adjustment factor for right turn in lane group

$f_{L T}=$ adjustment factor for left turn in lane group

$f_{L p b}=$ pedestrian adjustment factor for left turn movements

$f_{\text {Rpb }}=$ pedestrian-bicycle adjustment factor for right turn movement
Using Eq. (2), the base SFR, and the adjustment factors can be substituted to estimate the SFR of a lane group.

A study conducted in 2007 (Long, 2007) showed that an ideal SFR is not the same for different locations. Long (2007) stated that the concept in the HCM of the base saturation flow rate being constant implies an underlying model that saturation flow rate is equal to capacity, without the involvement of other variables. Several jurisdictions have since undertaken the effort to determine a default SFR other than the one recommended by the HCM, which is more in line with local conditions.

Bonneson et al. (2005) conducted a study to determine the ideal SFR for through lane movements and developed adjustment factors that account for the effects of area type, number of lanes and presence of rightturn vehicles at signalized intersections in Florida. The study was conducted at 35 intersections based on area type, right- 
turn volume, the number of lanes and area population. Different effects of adjustment factors on saturation flow rate were analyzed. The adjustment factors covered right-turn vehicle percentage, lane type, area population, the number of lanes, heavy vehicle percentage, traffic pressure, and speed limit. The results showed a weighted average saturation headway of $1.85 \mathrm{~s} / \mathrm{veh}$ and a corresponding base saturation flow rate of $1,950 \mathrm{pc} / \mathrm{h} / \mathrm{ln}$. The base rate of 1,950 $\mathrm{pc} / \mathrm{h} / \mathrm{ln}$ and the adjustment factors were recommended to be used by the state of Florida.

The Pennsylvania Department of Transportation (PENNDOT) District 12-0 (Dunlap, 2005) conducted a study in 2005 to determine the appropriateness of lower ideal SFR established by field measuring a sample of SFRs, from which the ideal SFR was computed. The ideal SFR used by District $12-0$ was 1,800 pcphgpl. The data collection was performed according to HCM procedures. Data were collected for through, left-turn and right-turn lane groups at five intersections in four different counties in southwestern Pennsylvania. The comparisons of ideal SFRs by county, lane type, approach grade, lane width, percentage heavy vehicles, atmospheric conditions, and time of day were analyzed using a one-way ANOVA and Duncan's Test. The study concluded that an ideal SFR of 1,800 pcphgpl used in District 12-0 was ideal. However, the study determined a weighted average ideal SFR 1,701 pcphgpl for the intersections studied.

A SFR study was conducted at 25 signalized intersections in Panama City, Republic of Panama in 2006 (Lewis and Benekohal, 2006). The purpose of the study was to determine the saturation headway and saturation flow rate in Panama City. The study focused on only through lanes while considering the effect of heavy vehicles with variations in lane widths on saturation headways. The percentages of heavy vehicles considered were $0,10,20,30$ and 40. Forty cycles for each site were used for the statistical analysis at $95 \%$ confidence level. Field data collection for headways were conducted using the standard HCM procedures based on which a representative SFR and saturation headway for the 25 intersections were calculated. The results of the analysis showed that the saturation headways from the field data were less than the default saturation headway of 1.9 seconds. The SFR for the 25 intersections were also calculated using the field data that showed that the default HCM value of 1,900 pcphplpg was not adequate for Panama City. A greater saturation flow rate was determined for Panama City traffic conditions. A twoway analysis of variance was also conducted to evaluate the effect of heavy vehicles and lane widths over the saturation headways, at a $5 \%$ level of significance. The results showed that the influence of heavy vehicles and lane width on saturation headway in Panama City was statistically significant while the interaction effect between heavy vehicles and lane width was also determined to be statistically significant.

In 2009, Zang and Chen in Nanjing, China used linear regression analysis to calibrate saturation flow rate for intersections based on data obtained at nine intersections. The study focused on the impact of heavy vehicles on saturation flow rate; other factors were not considered for this research. Therefore, it was assumed that:

$s=s_{O} f_{H V}$ 
where:

$s=$ saturation flow rate for subject lane group (vphgpl)

$S_{o}=$ ideal saturation flow rate per lane $(\mathrm{pc} / \mathrm{h} / \mathrm{ln})$

$f_{H V}=$ adjustment factor for heavy vehicles in traffic stream

The results showed that heavy vehicles have a high correlation with the average headway. Fig. 2 shows the resulting linear regression analysis model, where $t$ is the average time headway (seconds) and $P$ is the percentage of heavy vehicles (\%). An $F$-test was used to confirm the significance of the regression model with a significance level of $1 \%(0.01)$. An ideal saturation flow rate of $2,121 \mathrm{pc} / \mathrm{h} / \mathrm{ln}$ was determined for the intersections based on the resulting regression model.

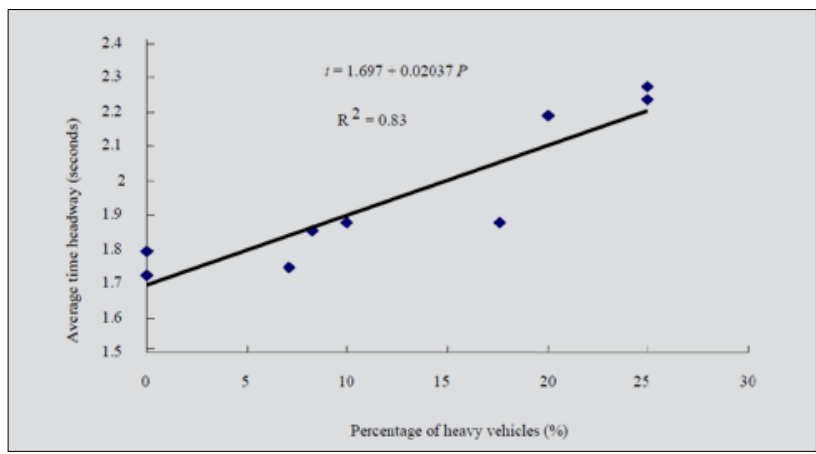

Fig. 2.

Regression Model for Average Headway and Percentage of Heavy Vehicles

Source: Zhang and Chen (2009)

Potts et al. (2007) determined a relationship between lane width and SFR based on data collected at 25 signalized intersections. The critical component of the study was to determine the ideal SFR using lane width adjustment factor. The study considered 2,733 vehicles for lane widths $8.5 \mathrm{ft}$. to 9.5 $\mathrm{ft}$., and 1,568 vehicles for lane width $13 \mathrm{ft}$. to $15.5 \mathrm{ft}$. The study assumed that all the adjustment factors were constant while varying only the lane width adjustment factor. The results showed that lane widths influenced the SFR values. In particular, the following average saturation flow rate ranges for specific lane widths were obtained at 5\% level of significance: $1,736-1,752 \mathrm{pc} / \mathrm{h} / \mathrm{ln}$ for $9.5 \mathrm{ft}$. lanes; $1,815-1,830 \mathrm{pc} / \mathrm{h} / \mathrm{ln}$ for 11 to 12 -ft lanes; and $1,898-1,913 \mathrm{pc} / \mathrm{h} / \mathrm{ln}$ for lane widths of $13 \mathrm{ft}$. or greater. A revision of the HCM lane with an adjustment factor for $12 \mathrm{ft}$.-lane was recommended.

The literature suggests that driver behavior also influences the value of the ideal saturation flow rate at intersections. Bonneson et al. (2005) deduced from their study that unsteady flow in otherwise highvolume conditions could reflect the presence of aggressive drivers. Drivers achieve this by accepting relatively short headways during queue discharge. Dunlap (2005) also proved that ideal SFRs are higher due to the presence of more aggressive drivers. Long (2007) developed a driver-behavior 
model that offers evidence that the default SFR is not likely constant in many situations, especially when a significant number of aggressive drivers are in the traffic stream.

In summary, the literature showed a wide variation of base $S F R$ values that are due to several factors, including intersection location, aggressive drivers, lane widths, and jurisdiction, among others. Therefore, the casual use of HCM's default SFR in the conduct of level of service analyses, especially for operational purposes, may skew the results and impair some traffic safety, operations and planning decisions. Thus, there is a need to determine a base SFR for each type of lane group by first determining the prevailing SFR.

\section{Research Methodology}

\subsection{Selected Sites for SFR Data Collection}

\subsubsection{Through Lane Group (T)}

Sixty through lane group (T) signalized intersections were identified for this study. They were selected based on their location in 6 zones with different characteristics previously defined by DDOT. Zone 5 represents the Central Business District (CBD) or downtown of the District of Columbia. The remaining zones (1-4 and 6; or radial areas) feed into Zone 5 . The majority of the intersections were located in the Central Business District in Washington, DC and covered various types of intersections and lane groups.

\subsubsection{Shared Through and Right Lane Group (TR)}

Fifteen (15) intersections were selected for the shared through and right- lane group.
These intersections were selected based on the availability of traffic volumes and queue lengths.

\subsubsection{Shared Through and Left- Lane Group (TL)}

Five (5) intersections were selected for the shared left-turn and through lane group. These locations were selected based on the signal phase sequence and the operation of the left turn movement. Only lead protectedpermitted TL movements were considered.

\subsubsection{Exclusive Left-turn Lane Group (L)}

Six (6) locations were selected for the exclusive left-turn lane group. These locations were also selected based on the lead signal phase sequence and the protectedpermitted operation mode of the turn movement.

\subsection{Data Collection}

Vehicle discharge headway observations for $\mathrm{T}, \mathrm{TR}, \mathrm{TL}$, and $\mathrm{L}$ lane groups at the 81 intersections were conducted during the morning (7:30 A.M. to 10:30 A.M.) and evening (3:30 P.M. to 6:30 P.M.) peak hours on weekdays from Monday to Friday. The field data required determining the prevailing SFRs for each lane group by using the procedures described in the HCM. The data collection process was conducted at the beginning of the green indication for the $\mathrm{T}$ and TR lane groups. For the TL and L lane groups data collection started at the beginning of the green ball with a green directional arrow. For each lane group, the lane observed to have the longest queue was selected for observation. Using stopwatches, the time $\left(t_{4}\right)$ was recorded when the rear axle of the fourth vehicle crossed the stop 
bar of the approach. The time $\left(t_{n}\right)$ was also recorded when the last vehicle in the queue at the beginning of green light crossed the stop bar. The total number $(N)$ of vehicles stopped in the queue at the beginning of the green was also recorded. Based on the HCM recommendation, a minimum of 15 cycles were observed to achieve a representative statistical sample. At least eight vehicles were observed in a queue before the data was recorded. Otherwise, the data for that cycle was ignored. The average headway was then computed by Eq. (4):

$\bar{h}=\frac{t_{n}-t_{4}}{N-4}$

\subsection{Data Analysis}

\subsubsection{Saturation Flow Rate}

When measured in the field, the prevailing SFR have units of vehicles per hour per lane. This can be computed using the following formula:

$s=3600 / h$

where

$s=$ saturation flow rate (vphpl)

$h=$ average time headway (sec/veh)

The following descriptive statistical summaries were computed for all the intersections in each lane group for the morning and evening peak periods:

- Average headway,

- Mean SFR,
- Standard deviation (of the SFR),

- $\quad 95 \%$ confidence interval (of the SFR).

\subsubsection{Test of Hypothesis}

The proposed hypothesis is that there is at least a significant difference between the average prevailing SFR for T, TR, TL and $L$ lane groups. A one-way ANOVA was used to test the hypotheses and is expressed mathematically as:

$H_{o}: \mu_{T}=\mu_{T L}=\mu_{T R}=\mu_{L}$

$H_{a}: \mu_{T} \neq \mu_{T L} \neq \mu_{T R} \neq \mu_{L}$

where $\mu_{T}, \mu_{T L}, \mu_{T R}$ and $\mu_{L}$ are the average prevailing SFRs for exclusive through, shared through and left-turn, shared through and right-turn, and exclusive left-turn lane groups respectively. A $95 \%$ confidence interval was used to test the hypothesis with the assumption that the sample is normally distributed. If the associated $p$-value is determined less than $(\alpha=0.05)(p$-value $<\alpha)$, we fail to accept the null hypothesis. However, if the $p$-value is greater than $\alpha$ ( $p$-value $>\alpha$ ), we fail to reject the null hypothesis. This test was conducted due to the fact that the magnitude of base SFR cannot be assumed equal for all lane groups used in this study.

\section{Results}

\subsection{Average Headways}

Table 1 presents the summary of the average headways for each lane group which were used to calculate the prevailing SFR. 
Table 1

Summary of Mean Average Headways by Lane Group

\begin{tabular}{|l|c|c|}
\hline \multirow{2}{*}{ Lane Groups } & \multicolumn{2}{|c|}{ Mean Average Headway } \\
\cline { 2 - 3 } & AM & PM \\
\hline Through & 2.3 & 2.4 \\
\hline Through/Right & 2.1 & 2.5 \\
\hline Through/Left & 3.0 & 2.5 \\
\hline Left & 2.4 & 2.6 \\
\hline
\end{tabular}

\subsection{Through Lane Group}

The summary of the descriptive statistics for the prevailing SFR by zone for through lane only is presented in Table 2 . From the table, the highest mean prevailing SFR was determined to be 1,692 vphpl for the intersections in Zone 2, while the lowest was determined to be $1,523 \mathrm{vphpl}$ in Zone 5 during the A.M. peak hour. The highest and lowest values of the mean prevailing
SFRs in the P.M. peak hour were determined to be respectively 1,613 and $1,480 \mathrm{vphpl}$, which were in Zones 3 and 5 respectively. For the 60 intersections, the mean prevailing SFR for both A.M. and P.M. peak periods were respectively 1,577 and 1,542 vphpl, corresponding to an overall mean prevailing SFR of $1,559 \mathrm{vphpl}$ for all the intersections. At $95 \%$ confidence interval, this overall average prevailing SFR value falls between 1,363 and 1,755 vphpl.

Table 2

Summary of Prevailing SFR by Zones for Through Lane Group

\begin{tabular}{|c|c|c|c|c|c|c|}
\hline \multirow{2}{*}{ Zone } & \multicolumn{2}{|l|}{$\mathbf{A M}$} & \multicolumn{2}{|l|}{$\mathbf{P M}$} & \multirow{2}{*}{$\mathbf{N}$} & \multirow{2}{*}{ Overall Average } \\
\hline & Mean & ST. DEV & Mean & ST. DEV & & \\
\hline 1 & 1,570 & 140.6 & 1,565 & 314.8 & 9 & 1,567 \\
\hline 2 & 1,692 & 141.7 & 1,558 & 91.6 & 8 & 1,625 \\
\hline 3 & 1,612 & 153.6 & 1,613 & 203.3 & 10 & 1,612 \\
\hline 4 & 1,664 & 270.1 & 1,545 & 260.4 & 4 & 1,605 \\
\hline 5 & 1,523 & 193.8 & 1,480 & 222.6 & 20 & 1,501 \\
\hline 6 & 1,524 & 83.2 & 1,563 & 209.8 & 9 & 1,544 \\
\hline
\end{tabular}

\subsection{TR, TL and L Lane Groups}

Table 3 presents the summary of the average prevailing SFR for the TR, T and L lane groups. From Table 3, the shared TL lane group has the highest prevailing SFR for both time periods $(1,544 \mathrm{vphpl}$ and 1,588 vphpl during the morning peak hour and evening peak hour respectively). The morning peak hour has a higher prevailing SFR than the evening peak hour for the lane groups except for the shared TL lane group, which has a higher prevailing SFR during the evening peak hour and at the same time, has the highest overall mean prevailing SFR of 1,566 vphpl. 
Table 3

Summary of Average Prevailing SFRs per Lane Group

\begin{tabular}{|c|c|c|c|c|c|c|c|c|c|}
\hline \multirow[b]{2}{*}{ Lane Group } & \multicolumn{4}{|l|}{ A.M. } & \multicolumn{4}{|l|}{ P.M. } & \multirow[b]{2}{*}{$\begin{array}{l}\text { Average } \\
\text { SFR }\end{array}$} \\
\hline & $\begin{array}{l}\text { Mean } \\
\text { SFR }\end{array}$ & $\mathbf{N}$ & ST. DEV & 95\% C.I & $\begin{array}{l}\text { Mean } \\
\text { SFR }\end{array}$ & $\mathbf{N}$ & ST. DEV & 95\% C.I & \\
\hline Through/Right & 1,495 & 15 & 143.7 & $1,442-1,606$ & 1,426 & 15 & 166.2 & $1,288-1,478$ & 1,461 \\
\hline Through/Left & 1,544 & 5 & 231.8 & $1,220-1,684$ & 1,588 & 5 & 209.7 & $1,377-1,797$ & 1,566 \\
\hline Exclusive Left & 1,470 & 6 & 170.3 & $1,282-1,623$ & 1,450 & 6 & 196.2 & $1,263-1,655$ & 1,460 \\
\hline
\end{tabular}

\subsection{Comparing Average Prevailing SFRs}

the highest mean prevailing SFR value with 1,577 vphpl, while the exclusive Left-turn

Fig. 3 presents the mean prevailing SFR lane had the lowest $(1,451 \mathrm{vphpl})$. Also, the values by lane group in the morning peak TL lane group recorded a mean prevailing hours. From the figure, the T lane group had SFR of 1,533 vphpl.

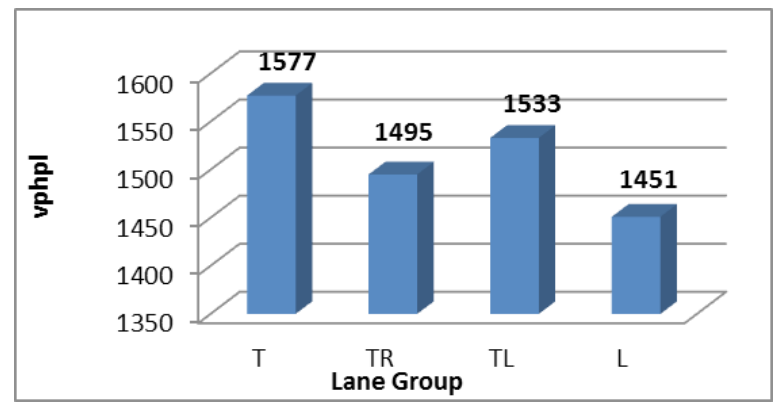

Fig. 3.

Mean Prevailing SFR by Lane Groups during the AM Peak Hour

The mean prevailing SFRs by lane groups vphpl, followed by the T lane group $(1,542$ during the evening peak are presented in Fig. vphpl). Again, the TR lane group had the 4. It can be observed that TL lane group had lowest mean prevailing SFR of 1,426 vphpl the highest mean prevailing SFR of 1,518 followed by the L lane group with 1,503 vphpl.

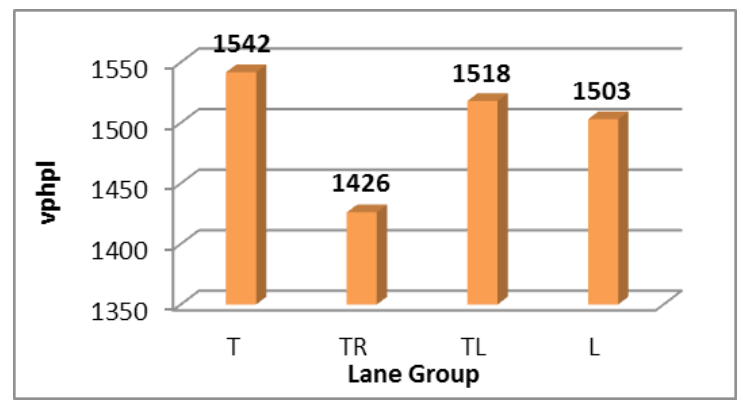

Fig. 4.

Mean Prevailing SFR by Lane Groups during the PM Peak Hour 


\subsection{One Way: Single Factor}

Tables 4 to 7 present the summary statistics and ANOVA for the morning and evening periods. The results show that the mean prevailing SFR for all 4 lane groups are statistically similar since $p>0.05$.

As a consequence, we fail to reject the null hypothesis at $5 \%$ level of significance.

\section{Table 4}

Summary Statistics - AM

\begin{tabular}{|l|l|l|l|l|}
\hline Groups & Count & Sum & Average & Variance \\
\hline T & 60 & 94,604 & $1,576.7$ & 29367.1 \\
\hline T/R & 15 & 22,427 & $1,495.1$ & 22115.3 \\
\hline T/L & 5 & 7,718 & $1,543.6$ & 67174.8 \\
\hline $\mathbf{L}$ & 6 & 8,819 & $1,469.8$ & 34781.0 \\
\hline
\end{tabular}

\section{Table 5}

ANOVA Single Factor - AM

\begin{tabular}{|l|l|l|l|l|l|}
\hline Source of Variation & SS & MS & F & P-value & F critical \\
\hline Between Groups & 125965.3 & 41988.45 & 1.386 & 0.253 & 2.716 \\
\hline Within Groups & 2484874 & 30303.34 & & & \\
\hline Total & 2610839 & & & & \\
\hline
\end{tabular}

\section{Table 6}

Summary Statistics - PM

\begin{tabular}{|l|l|l|l|l|}
\hline Groups & Count & Sum & Average & Variance \\
\hline T & 60 & 92,509 & 1541.8 & 48457.9 \\
\hline T/R & 15 & $21,394.3$ & 1426.3 & 29588.1 \\
\hline T/L & 5 & 7,938 & 1587.6 & 54974.8 \\
\hline $\mathbf{L}$ & 6 & 8,698 & 1449.7 & 46212.3 \\
\hline
\end{tabular}

\section{Table 7}

ANOVA Single Factor - PM

\begin{tabular}{|l|l|l|l|l|l|}
\hline Source of Variation & SS & MS & F & P-value & F critical \\
\hline Between Groups & $2 \mathrm{E}+05$ & 70811.8 & 1.55 & 0.20 & 2.71 \\
\hline Within Groups & $4 \mathrm{E}+06$ & 45417.1 & & & \\
\hline Total & $4 \mathrm{E}+06$ & & & & \\
\hline
\end{tabular}

\section{Discussion of Results}

According to the $2010 \mathrm{HCM}$, determining the prevailing SFR is the first step in the determination of a local base SFR for a local jurisdiction. The prevailing SFR is adjusted to and combined with different factors to determine a local base SFR. A local base 
SFR can be used to accurately determine the existing LOS and control delay of an intersection.

From the results, the highest mean prevailing SFR for the through lane group was computed to be approximately $1,692 \mathrm{vphpl}$, while the lowest was 1,523 vphpl during the A.M. peak hour. The highest and lowest values of the mean prevailing SFRs in the P.M. peak hour were determined to be 1,613 and $1,480 \mathrm{vphp} 1$ respectively. For the 60 locations observed for the through lane group, the overall mean SFR was approximately $1,560 \mathrm{vphpl}$, which falls between 1,363 and 1,755 vphpl at a $95 \%$ confidence interval.

Also, the through lane group had the highest prevailing SFR of $1,577 \mathrm{vphpl}$ during the morning peak hour, while the shared leftturn and through lane had the highest prevailing SFR of $1,518 \mathrm{vphpl}$ during the evening peak hour. The mean prevailing SFR were generally higher during the A.M. peak hour for all the lane groups except the L lane group, which had a higher prevailing SFR during the evening peak hours. At 5\% level of significance, the mean prevailing SFRs for all the lane groups considered in this study are statistically equal.

\section{Conclusions and Recommendations}

Based on the outcome of this study, the local base SFR for signalized intersections can be determined following the process prescribed in the HCM. The adjusted SFR can be computed for each lane group in the data set. Using the average prevailing SFR determined in this study, the local base SFR can then be computed using the following formula:

$$
S_{o, \text { local }}=\frac{1,900 \sum_{i=1}^{m} S_{\text {prevailing }, i}}{\sum_{i=1}^{m} S_{i}}
$$

where

$S_{o, \text { local }}$ local base saturation flow rate $(\mathrm{pch} / \mathrm{ln})$

$S_{\text {prevailing, } i=}$ prevailing SFR for lane group $i$ (veh/h/ln)

$S_{i}=$ adjusted SFR for lane group I (veh/ $\mathrm{hr} / \mathrm{ln})$ and

$m=$ number of lane groups

The computed local base SFR can be used for the purposes of computing average vehicular delay per lane group and thereby the overall level of service for the intersection.

The study concluded that the prevailing SFR values for the $T, T R, T L$, and $L$ lanes groups were statistically similar.

\section{Acknowledgements}

The authors acknowledge the District Department of Transportation for funding and supporting this study.

\section{References}

Bonneson, J.; Nevers, B.; Zegeer, J.; Nguyen, T.; Fong, T. 2005. Guidelines for Quantifying the Influence of Area Type and Other Factors on Saturation Flow Rate. Florida Department of Transportation and Texas Transportation Institute, College Station, Texas.

Dunlap, B.M. 2005. Field Measurement of the Ideal Saturation Flow Rate. From the Highway Capacity Manual, West Virginia University, Morgantown, West Virginia.

Lewis, E.E.; Benekohal, R.F. 2006. Saturation Flow Rate Study at Signalized Intersection in Panama: Transportation Research Board 2007 Annual Meeting, 07-3464.

Long, G. 2007. Variability in Base Saturation Flow Rate. Paper No. 07-2689. In 86th Annual Meeting Compendium of Papers CD-ROM, TRB, National Research Council, Washington, DC. 
Potts, I.B.; Bauer, K.M.; Harwood, D.W.; Gilmore, D.K. 2007. Relationship of Lane Width to Saturation Flow Rate on Urban and Suburban Signalized Intersection Approaches, Journal of the Transportation Research Board, 2027: 45-51.

Transportation Research Board National Research Council. Highway Capacity Manual. 2010. Washington D.C.: TRB 2000. 4-7 to 4-12, 18-35 to18-36.

Zhang, G.; Chen, J. 2009. Study on Saturation Flow Rates for Signalized Intersections. In Proceedings of the International Conference on Measuring Technology and Mechatronics Automation, Southeast University, Nanjing China. 